Title:

\title{
Think Different with RNA Therapy: Can Antisense Oligonucleotides Be Used to Inhibit Replication and Transcription of SARS-CoV-2?
}

\section{Running title}

SARS-CoV-2 knock-down with antisense oligonucleotides

Eric Barrey ${ }^{1}$, Veronica Burzio ${ }^{2}$, Sophie Dhorne-Pollet ${ }^{1}$, Jean-François Eléouët ${ }^{3}$, Bernard Delmas ${ }^{3}$

Affiliations:

1 Université Paris-Saclay, INRAE, AgroParisTech, GABI, 78350 Jouy-en-Josas, France.

2 Department of Biological Sciences, Faculty of Life Sciences, Universidad Andrés Bello Fundación Ciencia \& Vida, Andes Biotechnologies SpA, Santiago, Chile

3 Université Paris-Saclay, INRAE, AgroParisTech, UMR VIM, Virologie et Immunologie Moléculaire, 78350 Jouy-en-Josas, France.

\section{Corresponding authors:}

Eric Barrey: eric.barrey@inrae.fr

Bernard Delmas: bernard.delmas@inrae.fr

Version: 01/05/2020 


\section{Abstract}

The severity of the global COVID-19 pandemic caused by SARS-CoV-2, with a high transmission rate, $2.6-4.7 \%$ lethality and a huge economic impact, poses an urgent need for efficient therapies and vaccines. Currently, there are only non-specific treatments to assist patients in acute respiratory distress during the inflammatory response that follows SARS-CoV-2 viremia. Drug repurposing clinical trials have been quickly launched at the international level. Specific treatments such as plasma transfusion from recovered patients into infected patients or the use of specific inhibitors of the viral RNApolymerase complex are promising strategies for blocking infection. To complete the therapeutic arsenal, we believe that the opportunity of targeting the SARS-CoV-2 genome by RNA therapy should be deeply investigated. In the present paper, we propose to design antisense oligonucleotides targeting transcripts encoding viral proteins associated to replication and transcription of SARS-CoV-2, aiming to block infection.

We designed antisense oligonucleotides specifically targeted to the genomic 5' untranslated region (5'-UTR), open reading frames $1 \mathrm{a}$ and $1 \mathrm{~b}$ (ORF1a and ORF1b), which govern the expression of the replicase/transcriptase complex, and gene N, which encodes the genome-associated nucleoprotein. To maximize probability of efficiency, we predicted the antisense oligonucleotides by using two design methods: i) conventional antisense oligonucleotides with $100 \%$ phosphorothioate modifications (ASO); ii) antisense locked nucleic acids (LNA) GapmeRs. After binding the viral RNA target, the hetero-duplexes (antisense oligonucleotide-viral RNA) should be cleaved by RNase H1. Nine potent ASO candidates were found and we selected four of them targeting ORF1a (1), ORF1b (2) and N (1). Nine GapmeR candidates were predicted with excellent properties and we retained four of them, targeting 5'-UTR (1), ORF1a (1), ORF1b (1) and 
N (1). The most potent GapmeR candidate targets the 5'-UTR, a key genomic domain with multiple functions in the viral cycle. By this open publication, we are pleased to share these in silico results with the scientific community in the hopes of stimulating innovation in translational research in order to fight the unprecedented COVID-19 pandemic. These antisense oligonucleotide candidates should be now experimentally evaluated.

Keywords:

coronavirus; COVID-19; antisense oligonucleotide; ASO; LNA GapmeR 


\section{Introduction}

Three coronaviruses have crossed the species barrier to cause deadly pneumonia in humans since the beginning of the $21^{\text {st }}$ century: severe acute respiratory syndrome coronavirus (SARS-CoV), Middle-East respiratory syndrome coronavirus (MERS-CoV), and SARS-CoV-2 (Walls et al., 2020 and references therein). The first SARS and MERS epidemics were rapidly contained, compared to the current SARS-CoV-2 outbreak, which emerged from Wuhan (China). SARS-CoV-2 has spread around the world, with over 2.2 million people infected (not entirely confirmed by RT-qPCR tests) and over 150,000 deaths, with a death rate of $0.2-14.7 \%$, in 185 countries (Johns Hopkins COVID19 data recorded on April 17th 2020 ). According to the early epidemiological reports in China, $63 \%$ of patients required hospitalization within 6-8 days after illness onset and $72 \%$ required transfer to intensive care units. Finally, $2.6-4.7 \%$ of the infected patients died (Zhou et al., 2020; Adhikari et al., 2020). In Europe, lethality has reached to date 1315\% in Belgium, United Kingdom, France and Italy (Johns Hopkins COVID-19 data recorded on April 19th 2020 ). Therefore, there is an urgent need to propose a panel of treatments for cases of varying severity. However, there is currently no specific treatment available. Numerous drug repurposing clinical trials have been launched around the world to treat SARS-CoV-2 infected patients. For example, Remdesivir, a novel antiviral drug in the class of nucleotide analogs, found to show antiviral activity against single-stranded RNA viruses including MERS and SARS viruses, is currently under study in several cohorts. Other clinical trials including drugs such as lopinavirritonavir, interferon $\beta$, or hydroxy-chloroquine alone or combined with the antibiotic azithromycin are currently being tested (European clinical trial Discovery; USA clinical $\underline{\text { trials; }}$;autret et al., 2020). The strategy of drug repurposing is a short-term response to 
the emergency but may not be helpful in finding effective treatments without severe adverse effects.

The use of anti-SARS2-CoV-2 antibodies from people who recovered to treat patients by plasmapheresis also represents a potent alternative to block viral replication. Plasma transfer is a well-established, but heavy procedure that has been used recently to treat Ebola patients. In the future, conferring protective immunity to the world population through the development of efficient vaccines will require at least 12 months (Wang et al., 2020). RNA vaccines represent a potent hope to produce safe vaccines in a more timely fashion than proteinic antigens or DNA vaccines.

To complete the therapeutics arsenal, antisense RNA therapy represents an emerging strategy to specifically treat various diseases. This technology is based on the use of antisense oligonucleotides (ASOs) targeted to mRNAs, small RNAs or long non-coding RNAs. ASOs enter cells, by as yet unclear mechanisms (Lobos-González et al., 2016), binds the RNA target, and this RNA strand present in the resulting double-stranded hetero-duplex (Antisense oligonucleotide - RNA Target) is cleaved by cellular RNase H1 (Wheeler et al., 2012). Antisense RNA therapy is an emerging topic of translational research in medicine and currently the focus of many clinical trials in cancers, myopathies, Huntington's disease and many other diseases (Crooke et al., 2018). For example, a phase $1 \mathrm{~b}$ clinical assay is currently under way for the treatment of solid cancers by an antisense oligonucleotide targeted to ASncmtRNA (Burzio et al., 2009; Lobos-González et al., 2016; Fitzpatrick et al., 2019; ClinicalTrials Identifier: NCT03985072). All these examples of ASOs in clinical trials show that the concept of antisense RNA therapy is coming of age and will be developed further in the near future thanks to its low toxicity, high specificity and low production cost. 
The principle of RNA silencing has been previously studied in vitro to curb the replication of highly pathogenic RNA viruses (reviewed in Spurgers et al., 2008; Kim et al., 1998; Li et al., 2015). Thus, considering previous experience in antisense RNA therapy, we hypothesize that this strategy could be applied as an anti-viral drug by binding to and cleaving SARS-CoV-2 RNAs. Considering that SARS-CoV-2 is an RNA virus which does not integrate into the host genome, this strategy could yield more efficient results than with retroviruses (Grillone and Henry, 2008).

The aim of this conceptual paper was to design specific antisense oligonucleotides to efficiently inhibit replication and transcription of the coronavirus SARS-CoV-2. The SARS-CoV-2 genome is a $30 \mathrm{~kb}$ positive sense RNA molecule that includes a 5'untranslated region (5'-UTR) of about 265 bases, followed by $22 \mathrm{~kb}$ encoding the replication and transcription machineries in ORF1a (13202 bases) and ORF1b (8086 bases) (Figure 1). The nucleoprotein N gene (1259 bases) is located at the 3'-end of the genome. These four genomic regions were analyzed using two predictive tools to design conventional ASOs and antisense locked nucleic acids (LNA) GapmeRs to block SARSCoV-2 replication and transcription.

\section{Methods}

The reference sequence of SARS-CoV-2 genomic RNA (GenBank MN908947 or Refseq NC_045512; Wu et al. 2020) was used to design the ASO and GapmeR candidates (Figure 1). In order to identify efficient antisense oligonucleotide candidates to knockdown the viral RNA fragments, we applied two validated tools commonly used for RNA silencing: i) conventional antisense oligonucleotides with $100 \%$ phosphorothioate modifications (ASO or PTO-modified ASO from here on); ii) antisense locked nucleic acids (LNA) 
GapmeR $^{\circledR}$ (GapmeR from here on). These two chemical modifications of antisense oligonucleotides are key options in oligonucleotide synthesis meant to obtain ASO or GapmeR stability and finally efficient cleavage of the viral RNA targets by RNase H1 (Figures S1 and S2).

Conventional ASO design: to predict the 15-20 nt long antisense oligonucleotides, we used the primer design tool from CLC Genomic Workbench v6 (CLC bio). The antisense oligonucleotides are synthesized with $100 \%$ phosphorothioate linkages (PTOmodification) by several biotechnology companies around the world (Eurofins, Integrated DNA Technology, BioSearch Inc. and others). PTO-modifications increase the stiffness of the backbone, and the stability and nuclease resistance of the antisense oligonucleotides (Figure S1-A). For each ASO candidate, several properties were computed and a synthetic score was obtained to sort the ASOs according to their predicted quality in RNA target binding. The algorithm for conventional ASO identification was used on 5,000 base stretches covering 5'-UTR, ORF1a, ORF1b and N. After selection of the best ASO candidates according to their molecular score and position on the viral fragments (5'UTR, ORF1a, ORF1b and N), we checked their specificity against the virus by searching putative off-targets by using perfect alignments (Megablast with highly similar sequences option) on the whole human transcriptome. ASO candidates that showed $100 \%$ on similar strand $+/$ - on the transcriptome were rejected even if their molecular score was high.

Antisense locked nucleic acids GapmeRs: these antisense oligonucleotides were initially developed by Exiqon and are now manufactured by Qiagen (LNA GapmeR®). GapmeRs are 14-16 nt long and include a combination of two oligonucleotide 
modifications: a 100\% phosphorothioate modified backbone (Figure S1-A) and some locked nucleic acids modifications (LNA) distributed along the sequence to increase stability and specificity (Figure S1-B; Bondensgaard et al., 2000). LNA modification is a bicyclic high affinity RNA mimic with the sugar ring locked in the 3'-endo conformation (Petersen et al., 2002). LNA nucleotides obey Watson-Crick base-pairing rules and stable A-helix with good base-stacking. This nucleotide modification increases the melting temperature (Tm; $+2-8^{\circ} \mathrm{C}$ per LNA base included in the sequence), confers nuclease resistance to oligonucleotides, increases their stability and potency in cells and finally provides high specificity to RNA target binding.

Antisense LNA GapmeRs are designed by a proprietary algorithm available on the Qiagen website. For each RNA target submitted, a score is computed to sort 10 GapmeR candidates. The putative off-targets of the designed GapmeRs on the reference human genome are checked to keep only the candidates without any off-targets. Then, the best GapmeR candidates are sorted into two categories «Excellent design» or «Good design ». In the present study, we kept only « Excellent design » with scores ordered from 1 to 4. Unfortunately, the algorithm cannot compute and compare all the GapmeR candidates on a long target sequence like 5'-UTR-ORF1a-ORF1b, which contains 21,555 nt. Thus, to compare the scores of the different GapmeR candidates between the viral fragments (5'-UTR, ORF1a, ORF1b and N), we undertook the design of GapmeR in two steps: first, we computed the design on each of the isolated fragments according to the annotations of the sequence (GenBank MN 908947); second, we computed the design on three chimeric sequences by fusing the 5'UTR (265 nt) successively with each fragment (ORF1a, ORF1b and N). Finally, the following targets were analyzed: 5'-UTR, ORF1a, ORF1b, N, 5'-UTR+ORF1a, 5'-UTR+ORF1b, 5'-UTR+N. Thus, the quality of the Gapmer candidates found on 5'UTR and the three other fragments was compared in order to 
retain the best candidates. In addition, we checked the reproducibility of the results on another SARS-CoV-2 sequence (GenBank MN988668).

\section{In silico validation of the antisense oligonucleotide candidates for targeting the}

SARS-CoV-2 sequences: we performed a strict complementary alignment (strand -/+) with all the SARS-CoV-2 genomes available on the GenBank database by using Betacoronavirus BLAST tool.

\section{Results}

The two algorithms predicted at least 18 ASO and GapmeR candidates with high scores located on the four target viral fragments (Figure 2). Table 1 shows the nine best-scored ASO candidates for targets 5'-UTR, ORF1a, ORF1b and $\mathrm{N}$ and more details on their molecular properties are shown in Table S1. We sorted the nine ASO candidates according to their molecular score and after rejecting those with perfect off-target alignments on the human transcriptome (100\% aligned strand +/-). Finally, we retained the four best ASO candidates in Table 1: one specific to ORF1a, two to ORF1b and one to fragment N. The 5'-UTR seems to be poorly targetable with ASO design since many offtarget hits were identified on the human transcriptome.

All the ASO candidates specifically target all the SARS-CoV-2 sequences available on the GenBank database with $100 \%$ complementary alignment (strand+/-) on each genome. Therefore, these ASOs will be synthesized with 100\% PTO linkages.

The design of the antisense LNA GapmeRs using the proprietary algorithm from Qiagen provided up to 10 GapmeR candidates per RNA target, all qualified as «Excellent design ». The two-step design of simple and chimeric sequences (5'-UTR-Other Fragment) allowed to sort the nine best GapmeR candidates (Table 2). The best candidate, designated GAP1, was found on the 5'-UTR in all the chimeric sequences. 
Three GapmeR candidates were identified in ORF1a and ORF1b, and two candidates on fragment N. No off-targets were found for these nine GapmeR candidates, due to the high specificity of the LNA GapmeR design feature. Finally, we selected the best GapmeR candidates: GAP1 on 5'UTR and three candidates scored 2 on each of the other fragments, ORF1a, ORF1b and N. In addition, we computed the GapmeRs on a second, recently published SARS-CoV-2 sequence (GenBank MN988668) and we found the best GapmeR candidates (scored 1-2) at the same positions (Figure S3). The GapmeR positions with the highest scores, 1-2, selected in Table 1, are well-conserved on the two viral genomes: GAP1 on 5'UTR, GAP2a on ORF1a, GAP2b on ORF1b and GAP2n on N stay similar in the two design predictions (Figure S3).

Finally, we checked that all the GapmeR candidates of Table 2 bound perfectly to all the SARS-CoV-2 sequences available on GenBank with 100\% complementary alignment (strand+/-) on each genome. Altogether, these results confirmed the robustness of our GapmeR predictions and the genetic stability of the targets chosen on the viral sequences. The four selected GapmeRs with high potency will be synthesized.

In a future study, the best selected ASO and GapmeR candidates should be tested in vitro by transfecting infected and control cells according to a protocol adapted from Vidaurre et al. (2014).

\section{Discussion}

In this paper, by using two dedicated algorithms, we identified a total of nine ASOs with phosphorothioate modification and nine antisense LNA GapmeR candidates. Among them, we selected four PTO-modified ASOs and four GapmeRs scored as the best candidates that have a high probability of binding SARS-CoV-2 RNA sequences and 
therefore blocking viral multiplication. Both design methods could be efficient and should be tested in in vitro experiments, using SARS-CoV-2-infected and control human cells. By using the LNA GapmeR design, we predicted a higher number of high-quality antisense candidates (10 per fragment) without off-targets, as compared to conventional ASO design. However, we can briefly speculate on the advantages and drawbacks of each type of design.

ASOs with phosphorothioate modifications are recognized as the most useful approach to date for RNA silencing and RNA therapy, with good pharmacokinetics of half elimination in 1-3 days, optimal protein binding and a lower melting temperature than oligonucleotides with normal phosphodiester linkages (Crooke et al., 2018). The ASO method, based on single-strand modified oligonucleotides has the advantage of targeting any RNA sequence in exons or introns before splicing. In addition, ASOs have a high capacity to be naturally absorbed by cells in vivo even by very highly differentiated cells such as neurons (Wild and Tabrizi, 2018). The specificity of the ASO design should be checked by the research of putative off-targets on the human transcriptome. For future therapeutic applications, it has been demonstrated in several clinical trials that subcutaneous injection of ASO up to $800 \mathrm{mg}$ are well-tolerated and ASOs at a high dose can be naturally delivered into cells (Clinical Trial ID NCT02508441).

Antisense LNA GapmeRs may be more stable, more specific and more efficient than ASOs in binding and cleaving viral RNA targets because the two combined LNA GapmeR modifications optimize the stability of the hetero-duplex that recruits RNase H1 (Figure S1; Wheeler et al., 2012). For in vivo therapeutic applications, another advantage of antisense LNA GapmeRs and PTO-modified ASOs is their high ability to be physiologically absorbed by cells via unassisted delivery (transfection-free) when the GapmeRs are injected at high dose and purity. Antisense LNA GapmeRs were 
successfully applied in vivo in a mouse PDX model of human melanoma to knockdown SAMMSON IncRNA, involved in translational activation of the tumor cells (Leucci et al., 2016). One drawback observed in a clinical trial was the reversible renal and hepatotoxicity observed in one of the clinical trials (Bianchini et al., 2013). In virology, a clinical trial demonstrated the acceptable safety and efficiency of an antisense LNA to reduce the level of hepatitis $\mathrm{C}$ virus (HCV) infection. Although some adverse events were observed, they didn't require stopping the treatment (Janssen et al., 2013). Another in vitro study successfully applied antisense LNA design to block HIV-1 RNA in infected cells and compared the results with other ASO technologies (Jakobsen et al., 2007).

Other types of oligonucleotide modifications have been used for experimental RNA silencing and in therapeutics. Morpholinos (phosphorodiamidate modification) were extensively used a few years ago for RNA silencing and therapeutic applications with high stability and tolerability but low protein-binding capacity. Several drugs approved by the FDA were based on this type of design (Crooke et al., 2018).

The great therapeutic advantage of the antisense oligonucleotides presented here (both PTO-modified ASOs and antisense LNA GapmeRs) is that they can be delivered in vivo to target cells without any transfection reagent. They are stable in sterile aqueous solution and can be administrated by subcutaneous injections. Interestingly, for a respiratory tract infection, LNA GapmeRs can be administrated by inhalation of an aerosol. Such aerosol can be produced by nebulization of the antisense oligonucleotide solution, as has been demonstrated in mouse models (Moschos et al., 2011). The advantages of this inhalation delivery would be lower toxicity and higher concentration of the antisense oligonucleotide reaching the pulmonary epithelium infected by SARS-CoV-2. 
Many preliminary studies in virology provided the first proof of the efficiency of antisense oligonucleotides to curb the replication of various viruses, such as SARS-CoV. Interestingly, Neuman and co-workers (2005) described potent peptide-conjugated antisense morpholino oligomers (P-PMO with a phosphorodiamidate modification) able to efficiently block SARS-CoV production in cultured cells at a concentration of $20 \mu \mathrm{M}$. Their strategy was to suppress viral replication by binding the conserved RNA elements implicated in viral RNA synthesis and translation. The most effective anti-SARS-CoV antisense P-PMO that was found targets the transcriptional regulatory sequence region present in the viral genomic 5'-UTR, with an inhibition of virus yield exceeding $10^{4}$-fold. It is important to note that it targets the same genomic region of SARS-CoV-2 as our predicted most potent GapmeR, suggesting that GAP1 candidate on 5'UTR may have a strong effect on SARS-CoV-2 replication.

\section{Conclusion and perspectives}

We predicted four conventional antisense (PTO-modified ASO) and four antisense LNA GapmeRs with a high potential to inhibit SARS-CoV-2 replication and transcription by inducing cleavage of the 5'-UTR, ORF1a, ORF1b or N domains of the viral RNA genome. By this open access publication, we would like to share with the scientific community the characteristics of these antisense oligonucleotide candidates, which need to be experimentally evaluated. We hope that in the next months, biotechnology companies can synthesize the antisense oligonucleotides predicted in the present study. Afterwards, virologists and RNA biologists should test in vitro and in vivo the efficiency of the ASO and GapmeR candidates to identify the best drug candidates in order to experimentally validate our in silico results. 
Many preliminary studies in virology provided the first proofs-of-concept of the efficiency of antisense nucleotides to curb the replication of several viruses such as SARS-CoV. Although the experimental validation may take several months and the clinical trial more than one year, such antisense RNA therapy targeting SARS-CoV-2, which seems to possess a stable genome, could be a valuable complement to a vaccine in the fight against the COVID-19 pandemic.

\section{Ethics statement}

This paper is a proposal of an antisense anti-viral RNA strategy which requires experimental validation in order to be entirely demonstrated. The authors are pleased to share these data but will have no responsibility in any misuse by other parties of the preliminary in silico results presented here. 


\section{Author contributions}

EB and BD developed the concept and wrote the paper.

VB contributed to the antisense RNA therapy strategy, wrote the paper with her expertise in ASO therapy in oncology.

SD-P contributed to the biomolecular and RNA engineering method.

JFE contributed with his expertise in coronavirus replication and transcription.

All authors reviewed the paper and provided final approval for the submitted version of the manuscript.

\section{Funding}

No specific funding except the usual financial support of our research institute INRAE.

\section{Conflict of interest}

The authors declare that the research was conducted in the absence of any commercial or financial relationships that could be construed as a potential conflict of interest.

\section{Acknowledgments}

The authors greatly acknowledged Mrs. Evelyne J. Barrey for the English revision.

\section{References:}

Adhikari, S. P., Meng, S., Wu, Y.-J., Mao, Y.-P., Ye, R.-X., Wang, Q.-Z., ... Zhou, H. (2020). Epidemiology, causes, clinical manifestation and diagnosis, prevention and control of coronavirus disease (COVID-19) during the early outbreak period: a scoping review. Infectious Diseases of Poverty, 9(1), 29. https://doi.org/10.1186/s40249-020-00646-X

Bondensgaard, K., Petersen, M., Singh, S. K., Rajwanshi, V. K., Kumar, R., Wengel, J., \& Jacobsen, J. P. (2000). Structural studies of LNA:RNA duplexes by NMR: conformations and implications for RNase H activity. Chemistry (Weinheim an Der Bergstrasse, 
Germany), 6(15), 2687-2695. https://doi.org/10.1002/15213765(20000804)6:15<2687::aid-chem2687>3.0.co;2-u

Bianchini, D., Omlin, A., Pezaro, C., Lorente, D., Ferraldeschi, R., Mukherji, D., ... Danila, D. C. (2013). First-in-human Phase i study of EZN-4176, a locked nucleic acid antisense oligonucleotide to exon 4 of the androgen receptor mRNA in patients with castrationresistant prostate cancer. British Journal of Cancer, 109(10), 2579-2586. https://doi.org/10.1038/bjc.2013.619

Burzio, V. a, Villota, C., Villegas, J., Landerer, E., Boccardo, E., Villa, L. L., ... Burzio, L. 0. (2009). Expression of a family of noncoding mitochondrial RNAs distinguishes normal from cancer cells. Proceedings of the National Academy of Sciences of the United States of America, 106(23), 9430-9434. https://doi.org/10.1073/pnas.0903086106

Crooke, S. T., Witztum, J. L., Bennett, C. F., \& Baker, B. F. (2018). RNA-Targeted Therapeutics. Cell Metabolism, 27(4), 714-739. https://doi.org/10.1016/j.cmet.2018.03.004

Fitzpatrick, C., Bendek, M. F., Briones, M., Farfán, N., Silva, V. A., Nardocci, G., ... Burzio, V. A. (2019). Mitochondrial ncRNA targeting induces cell cycle arrest and tumor growth inhibition of MDA-MB-231 breast cancer cells through reduction of key cell cycle progression factors. Cell Death \& Disease, 10(6), 423. https://doi.org/10.1038/s41419019-1649-3

Gautret, P., Lagier, J.-C., Parola, P., Hoang, V. T., Meddeb, L., Mailhe, M., ... Raoult, D. (2020). Hydroxychloroquine and azithromycin as a treatment of COVID-19: results of an open-label non-randomized clinical trial. International Journal of Antimicrobial Agents, 105949. https://doi.org/10.1016/j.ijantimicag.2020.105949

Jakobsen, M. R., Haasnoot, J., Wengel, J., Berkhout, B., \& Kjems, J. (2007). Efficient inhibition of HIV-1 expression by LNA modified antisense oligonucleotides and DNAzymes targeted to functionally selected binding sites. Retrovirology, 4, 1-13. https://doi.org/10.1186/1742-4690-4-29

Janssen, H. L. A., Reesink, H. W., Lawitz, E. J., Zeuzem, S., Rodriguez-Torres, M., Patel, K., ... Hodges, M. R. (2013). Treatment of HCV infection by targeting microRNA. New England Journal of Medicine, 368(18), 1685-1694. https://doi.org/10.1056/NEJMoa1209026

Kim, K. E., Salter, D. W., \& Dodgson, J. B. (1998). Examination of Antisense RNA and Oligodeoxynucleotides as Potential Inhibitors of Avian Leukosis Virus Replication in RP30 Cells. Poultry Science, 77(9), 1400-1410. https://doi.org/10.1093/ps/77.9.1400

Leucci, E., Vendramin, R., Spinazzi, M., Laurette, P., Fiers, M., Wouters, J., ... Marine, J.-C. (2016). Melanoma addiction to the long non-coding RNA SAMMSON. Nature, 531(7595), 518-522. https://doi.org/10.1038/nature17161

Li, H., Ying, T., Yu, F., Lu, L., \& Jiang, S. (2015). Development of therapeutics for treatment of Ebola virus infection. Microbes and Infection, 17(2), 109-117.

https://doi.org/10.1016/j.micinf.2014.11.012 
Lobos-González,.... \& Burzio, L. 0. (2016). Targeting antisense mitochondrial ncRNAs inhibits murine melanoma tumor growth and metastasis through reduction in survival and invasion factors. Oncotarget August.

Petersen, M., Bondensgaard, K., Wengel, J., \& Peter Jacobsen, J. (2002). Locked nucleic acid (LNA) recognition of RNA: NMR solution structures of LNA:RNA hybrids. Journal of the American Chemical Society, 124(21), 5974-5982. https://doi.org/10.1021/ja012288d

Moschos, S. A., Frick, M., Taylor, B., Turnpenny, P., Graves, H., Spink, K. G., ... Yeadon, M. (2011). Uptake, efficacy, and systemic distribution of naked, inhaled short interfering RNA (siRNA) and locked nucleic acid (LNA) antisense. Molecular Therapy, 19(12), 21632168. https://doi.org/10.1038/mt.2011.20

Neuman,B.W., Stein, D.A., Kroeker, A.D., Churchill, M.J., Kim,A.M.,Kuhn,P., Dawson, P., Moulton, H.M., Bestwick, R.K., Iversen, P.L., Buchmeier, M.J., 2005. Inhibition, escape, and attenuated growth of severe acute respiratory syndrome coronavirus treated with antisense morpholino oligomers. J. Virol. 79 (15), 9665-9676.

Spurgers, K. B., Sharkey, C. M., Warfield, K. L., \& Bavari, S. (2008). Oligonucleotide antiviral therapeutics: Antisense and RNA interference for highly pathogenic RNA viruses. Antiviral Research, 78(1), 26-36.

https://doi.org/10.1016/j.antiviral.2007.12.008

Vidaurre, S., Fitzpatrick, C., Burzio, V. a, Briones, M., Villota, C., Villegas, J., ... Burzio, L. O. (2014). Down-regulation of the antisense mitochondrial non-coding RNAs (ncRNAs) is a unique vulnerability of cancer cells and a potential target for cancer therapy. The Journal of Biological Chemistry, 289(39), 27182-27198.

https://doi.org/10.1074/jbc.M114.558841

Wang, N., Shang, J., Jiang, S., \& Du, L. (2020). Subunit Vaccines Against Emerging Pathogenic Human Coronaviruses. Frontiers in Microbiology, 11(February). https://doi.org/10.3389/fmicb.2020.00298

Wheeler, T. M., Leger, A. J., Pandey, S. K., MacLeod, A. R., Nakamori, M., Cheng, S. H., ... Thornton, C. A. (2012). Targeting nuclear RNA for in vivo correction of myotonic dystrophy. Nature, 488(7409), 111-115. https://doi.org/10.1038/nature11362

Wild, E. J., \& Tabrizi, S. (2018). Europe PMC Funders Group Therapies targeting DNA and RNA in Huntington 's disease, Lancet Neurol. 16(10), 837-847.

https://doi.org/10.1016/S1474-4422(17)30280-6.

Zhou, F., Yu, T., Du, R., Fan, G., Liu, Y., Liu, Z., ... Cao, B. (2020). Clinical course and risk factors for mortality of adult inpatients with COVID-19 in Wuhan, China: a retrospective cohort study. The Lancet, 6736(20), 1-9. https://doi.org/10.1016/s0140-

$\underline{\text { 6736(20)30566-3 }}$ 


\section{Web sites:}

COVID-19 Dashboard by the Center for Systems Science and Engineering (CSSE) at John Hopkins University and Medicine : https://coronavirus.jhu.edu/map.html

GenBank - NCBI data base of Covid-19 genomes:

https://www.ncbi.nlm.nih.gov/genbank/sars-cov-2-seqs/\#sra-sequences

Betacoronavirus BLAST against SARS-CoV-2 sequences of GenBank :

https://blast.ncbi.nlm.nih.gov/Blast.cgi?PAGE TYPE=BlastSearch\&BLAST SPEC=Betaco $\underline{\text { ronavirus }}$

Discovery clinical trial: https://www.inserm.fr/actualites-etevenements/actualites/covid-19-demarrage-essai-clinique-discovery

USA Clinical trials data bases:

https://clinicaltrials.gov/ct2/results?cond=\%22wuhan+coronavirus $\% 22$

Clinical Trial Phase I: Safety trial of one ASO targeting AS-IncmtRNA for solid cancer therapy:

https://clinicaltrials.gov/ct2/show/NCT02508441?term=Andes+Biotechnologies\&cond $=$ Cancer \&draw $=2 \&$ rank $=2$

Qiagen LNA GapmeR:

https://www.qiagen.com/fr/applications/rna-universe/gene-function-andsilencing/antisense-lna/ 


\section{Illustrations: Headings, Figures, Tables (see next pages)}

Figure 1: A. Schematic showing the concept of antisense RNA therapy applied to inhibit the replication and transcription of the SARS-CoV-2 RNA genome. Genomic sequences and ORFs associated to replication and transcription are indicated in green. The length of the corresponding ORFs are indicated (numbers of nucleotides) B. Schematic showing the genomic and subgenomic mRNAs (in red) that share identical 3' sequences and form a 3' nested set of RNAs. The genome is translated into two large polyproteins that are encoded by ORF1a and ORF1b, ORF1b being translated through a ribosomal frameshift. The $\mathrm{N}$ protein is encoded by the smallest mRNA. Polyprotein 1a and 1ab are processed and their products constitute the viral replicase and transcriptase machineries.

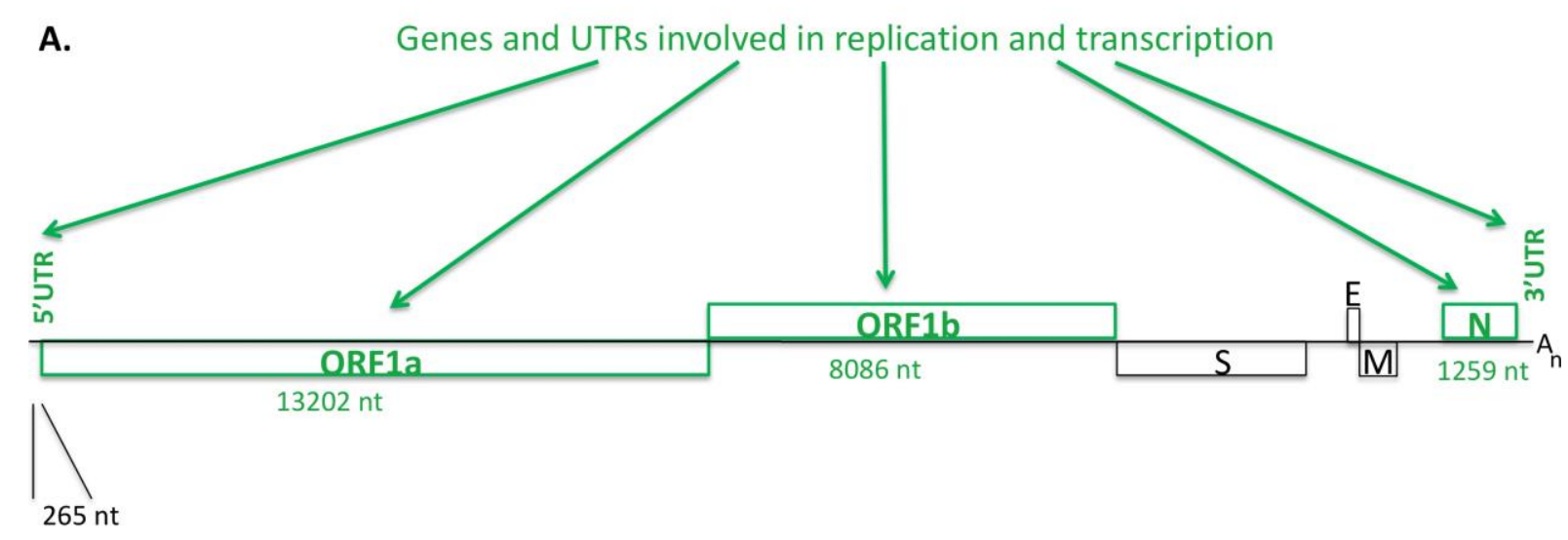

B.

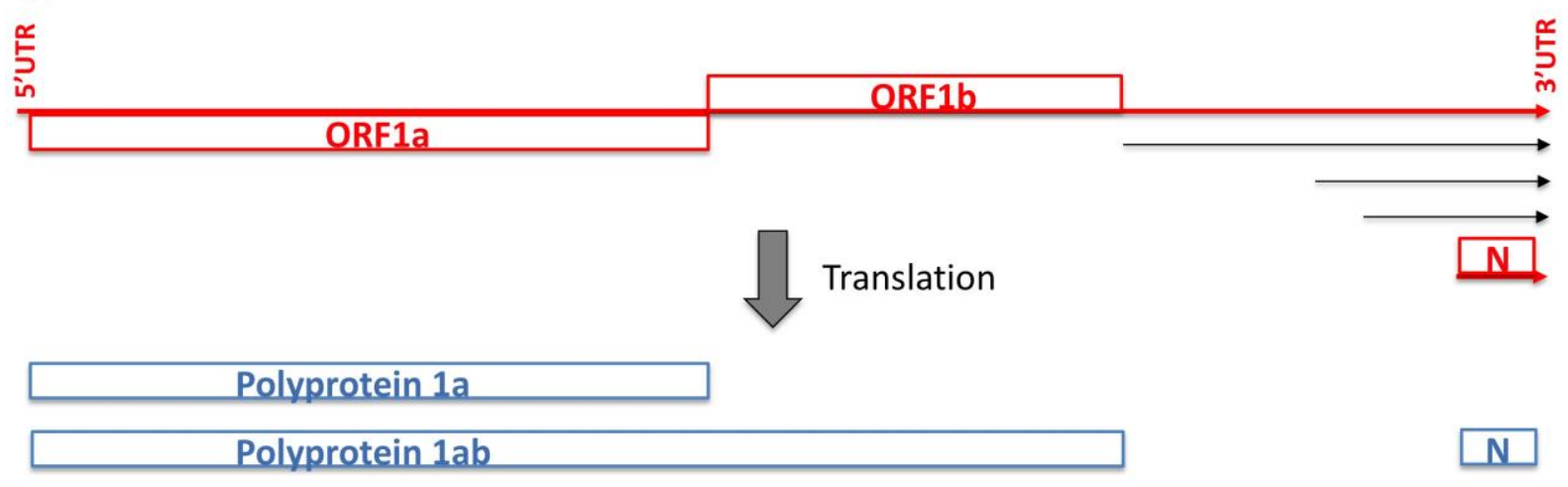


Figure 2: Positions of the best ASO and GapmeR candidates targeted to the 5'UTR, ORF1a ORF1b and N fragments. The ASOs and GapmeRs are listed in tables 1 and 2.

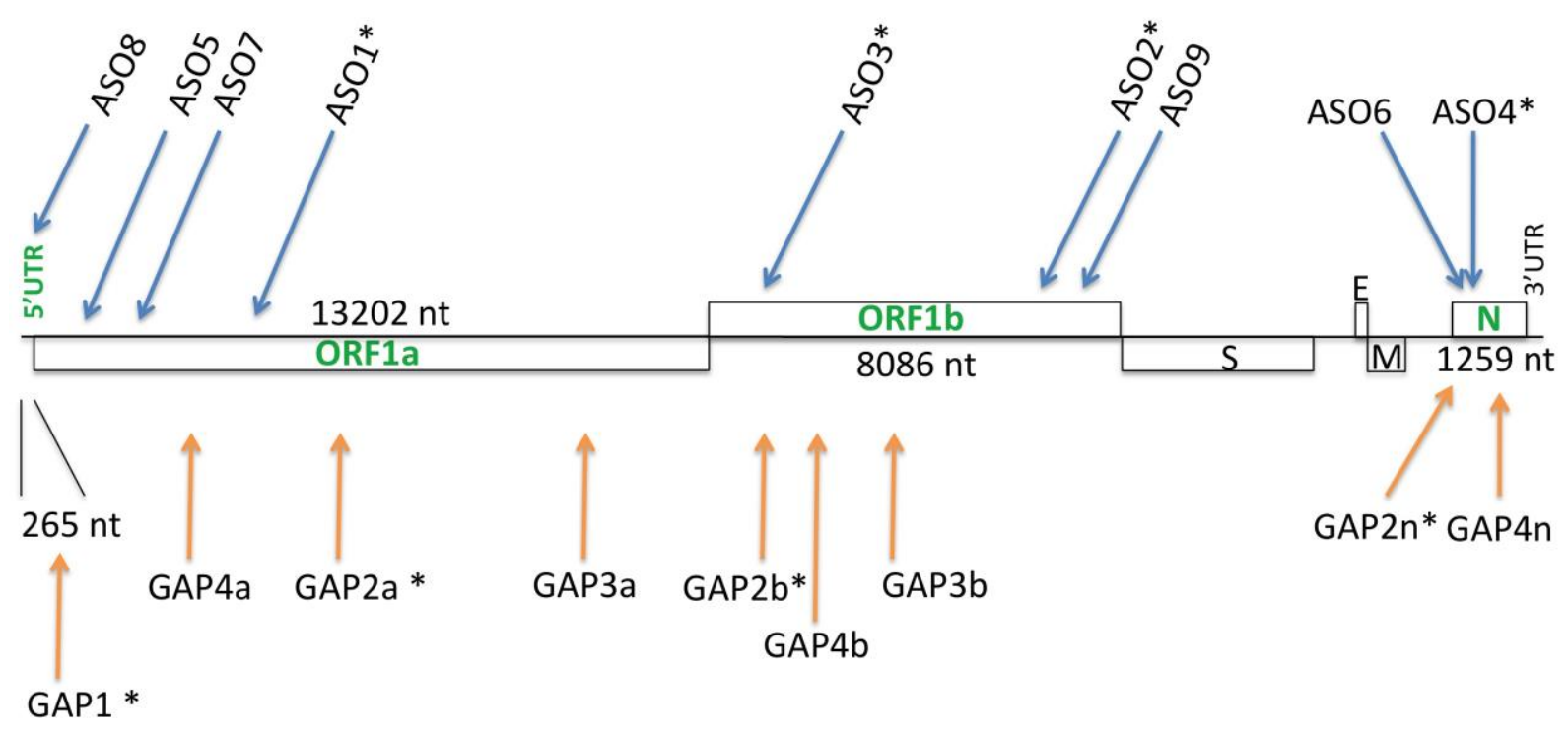


Table 1: ASO candidates to target 5'UTR, ORF1a, ORF1b and N fragments of the SARSCoV-2 genome (Genbank MN908947/RefSeq NC_045512.2). The ASOs are sorted according to their quality, including molecular score and potential off-targets on the human transcriptome. The best ASO candidates are marked by a star $\left({ }^{*}\right)$ according to the criteria explained in the Results section.

\begin{tabular}{|l|l|l|l|l|l|l|}
\hline ASO \# & $\begin{array}{l}\text { Fragment } \\
\text { target }\end{array}$ & Score & $\begin{array}{l}\text { Transcriptomic off- } \\
\text { targets } 100 \%\end{array}$ & & \\
\hline identical strands +/- & Position & Length & Sequence \\
\hline ASO1 * & ORF1a & 85.94 & no & $5917-5931$ & 15 & CAAACAACACCATCC \\
\hline ASO2 * & ORF1b & 83.31 & no & $20163-20177$ & 15 & ACAACACCATCAACT \\
\hline ASO3 * & ORF1b & 82.92 & no & $16457-16471$ & 15 & TGGGTGGTTTATGTG \\
\hline ASO4 * & N & 78.06 & no & $29108-29122$ & 15 & TTGGGTTTGTTCTGG \\
\hline ASO5 & ORF1a & 86.13 & 1 hit $>$ rejected & $1936-1950$ & 15 & AAAACACGCACAGAA \\
\hline ASO6 & N & 78.93 & 4 hits $>$ rejected & $28818-28832$ & 15 & AGGAACGAGAAGAGG \\
\hline ASO7 & ORF1a & 85.52 & 23 hits $>$ rejected & $3051-3066$ & 16 & CCTTCTTCTTCATCCT \\
\hline ASO8 & 5UTR & 94.10 & 20 hits $>$ rejected & $26-40$ & 15 & GTTGGTTGGTTTGTT \\
\hline ASO9 & ORF1b & 88.26 & 37 hits $>$ rejected & $20487-20501$ & 15 & ACAGAACACACACAC \\
\hline
\end{tabular}


Table 2: Antisense LNA GapmeR ${ }^{\circledR}$ candidates to target 5'UTR, ORF1a, ORF1b and N fragments of the SARS-CoV-2 genome (Genbank MN908947/RefSeq NC_045512.2). The GapmeRs are sorted according to their quality score within each fragment. All GapmeRs were classified as «Excellent design » by the prediction algorithm and have no offtargets. The best GapmeR candidates that we selected are marked by a star $(*)$ according to the criteria explained in the Results section.

\begin{tabular}{|l|l|c|l|l|l|l|l|}
\hline GapmeR \# & $\begin{array}{c}\text { Fragment } \\
\text { target }\end{array}$ & $\begin{array}{c}\text { Score } \\
\text { within } \\
\text { fragment }\end{array}$ & Design ID & Design name & Position & Length (nt) & Sequence \\
\hline GAP1 * & 5UTR & 1 & 901124 & 5P-1A-NC-30032020_1 & $32-47$ & 16 & ATCGAAAGTTGGTTGG \\
\hline GAP2a * & ORF1a & 2 & 901124 & 5P-1A-NC-30032020_2 & $7093-7108$ & 16 & GTAGGTTGCAATAGTG \\
\hline GAP2b * & ORF1b & 2 & 901125 & 5P-1B-30032020_2 & $16364-16379$ & 16 & TACGGATTAACAGACA \\
\hline GAP2n * & N & 2 & 901131 & 5P-N-NC-01042020_2 & $28420-28435$ & 16 & GGTGAACCAAGACGCA \\
\hline GAP3a & ORF1a & 3 & 901124 & 5P-1A-NC-30032020_3 & $10133-10148$ & 16 & GCCAAAGACCGTTAAG \\
\hline GAP3b & ORF1b & 3 & 901125 & 5P-1B-30032020_3 & $18253-18268$ & 16 & CGCGGGTGATAAACAT \\
\hline GAP4a & ORF1a & 4 & 901124 & 5P-1A-NC-30032020_4 & $3685-3700$ & 16 & AAGTAGAACTTCGTGC \\
\hline GAP4b & ORF1b & 4 & 901125 & 5P-1B-30032020_4 & $17134-17149$ & 16 & CTATGCGAGCAGAAGG \\
\hline GAP4n & N & 4 & 901131 & 5P-N-NC-01042020_4 & $28826-28841$ & 16 & TACGTGATGAGGAACG \\
\hline
\end{tabular}




\section{Supplementary materials}

Figure S1: Principle of antisense oligonucleotide activity for silencing viral RNA targets. Silencing by conventional PTO-modified ASOs and antisense LNA GapmeRs is based on the same RNase H-mediated cleavage mechanism.

Figure S2: Oligonucleotide modifications used in PTO-modified ASOs (A) and antisense LNA GapmeR (B) synthesis to increase their stability, resistance to nucleases and specificity.

Figure S3: Comparison of relative positions of the antisense LNA GapmeRs obtained on two different SARS-CoV-2 sequences, NC_045512.2 (MN908947) and MN988668. The candidates are scored from 1 (best) to 10 on each fragment.

Table S1: list of all ASO PTO-modified candidates, and their properties, targeted to 5'UTR, ORF1A, ORF1B and N fragment. 